\title{
Neoadjuvant chemotherapy versus cystectomy in management of stages II, and III urinary bladder cancer
}

\author{
Mohammed A. Osman ${ }^{1}$, Ayman M. Gabr ${ }^{2}$, Mohammad S. Elkady ${ }^{3}$ \\ ${ }^{1}$ General Organization for Teaching Hospitals Institutes, Egypt; \\ ${ }^{2}$ National Institute of Urology \& Nephrology, Egypt; \\ ${ }^{3}$ Ain Shams University, Egypt.
}

\begin{abstract}
Summary Purpose: This phase III trial was designed to compare the survival benefit, surgical respectability, and toxicities among patients treated by neoadjuvant chemotherapy followed by radical cystectomy (arm A), with those treated by radical cystectomy (arm B) in the management of stage II, III urinary bladder cancer.

Patients and Methods: For inclusion, patients should have pathologically proven urothelial carcinoma in urinary bladder, clinical stages from T2NOMO to T4aNOMO, patient age less than 65 years, and performance state $\leq 2$. Additionally, patients should have adequate hematological, renal, and liver functions. Arm A patients underwent 3 cycles of neoadjuvant cisplatin and gemcitabine followed by radical cystectomy, while arm B patients underwent radical cystectomy directly.

Results: Thirty patients had been enrolled in each arm between September 2009 and April 2014 in 3 educational institutes in Egypt. The 3 year OS (overall survival) for arm $A$, and $B$ were $60 \%$ and $50 \%$ respectively. The median OS for arm A was 36+ months and that for arm B was 32.5 months. The 3 year progression-free survival (PFS) for arm $A$, and $B$ were $57 \%$ and $43 \%$ respectively. The median PFS for arm $A$ was 36+ months and for arm $B$ was 28 months. A subgroup analysis was performed to correlate between 3 year OS and predetermined prognostic factors including age, tumor size, pathological stage, and the response to neoadjuvant chemotherapy. The later was performed only in arm A. Both treatment arms were tolerated well with mild toxicities profiles.

Conclusion: Neoadjuvant chemotherapy achieved better survival, surgical respectability, with nearly equivalent toxicities when compared with radical cystectomy.
\end{abstract}

KEY WORDS: Neoadjuvant; Cisplatin; Cystectomy; Survival; 3yOS; Arm A.

Submitted 14 July 2014; Accepted 30 September 2014

\section{BACKGROUND}

Bladder cancer is the ninth most common cancer throughout the world and is considerably more common in developing countries. Recently, it was shown that, incidence of transitional cell carcinoma (TCC) has been increasing while squamous cell carcinoma (SCC) has been decreasing. Active exposure to tobacco smoke has a strong relationship to TCC. Previous studies have reported a 2.6 fold risk of developing bladder cancer in smokers compared to nonsmokers (1).

Stage II bladder cancer refers to stages pT2aNOMO, where the tumor invades superficial muscularis propria, and pT2bNOMO, where it invades deep muscularis propria. Stage III refers to pT3NOMO, where the tumor invades perivesical tissue, and pT4aNOM0, where it invades prostatic stroma, uterus, vagina (2).

According to the recent NCCN guidelines, the standard management of stages II, and III bladder cancer included radical cystectomy, with strong consideration of neoadjuvant chemotherapy (3).

A trial conducted by the Medical Research Council and the European Organization for Research and Treatment of Cancer randomly assigned 976 patients with locally advanced (T3 or T4a) or high grade muscle-invasive (T2) bladder cancer to undergo either definitive treatment immediately or definitive treatment (surgery or radiotherapy) preceded by three cycles of neoadjuvant cisplatin, vinblastine, and methotrexate. At a median follow-up of 8.0 years, OS was significantly greater in the arm randomly assigned to receive neoadjuvant chemotherapy. The authors concluded that, the survival benefit from neoadjuvant chemotherapy conferred a $6 \%$ absolute increase in the likelihood of being alive at 3 years ( $56 \%$ vs. $50 \%), 5$ years ( $49 \%$ vs. $43 \%$ ), and 10 years (36\% vs. $30 \%$ ) (P 0.037) (4).

A meta-analysis of ten randomized trials of neoadjuvant chemotherapy, including updated data for 2,688 individual patients, showed that cisplatin-based combination chemotherapy was associated with a significant $13 \%$ relative reduction in the risk of death and resulted in 5\% improvement in 5-year survival from $45 \%$ to $50 \%$ (P 0.016) (5).

A subsequent meta-analysis of eight trials used multiagent, cisplatin-based chemotherapy, showed neoadjuvant chemotherapy was associated with a $6.5 \%$ absolute benefit in 5-year OS (50\% vs. 56.5\%) (P 0.006) (6).

Study objective was to compare the survival benefit, sur- 
gical resectability, and toxicities among patients treated by neoadjuvant chemotherapy followed by radical cystectomy with those underwent radical cystectomy in the management of stage $\mathrm{T} 2 \mathrm{NO}$, T3NO, and $\mathrm{T} 4 \mathrm{aNO}$ urinary bladder cancer. The primary end point was survival benefit.

\section{Patients and Methods}

Patients were eligible to be enrolled in the study if they had pathologically proven urothelial carcinoma in urinary bladder. Patients should have clinical stages from T2NOM0 to T4aNOM0 (as defined by CT scan) (7).

Patient age had to be less than 65 years and performance state had to be $\leq 2$. Patients should have adequate hematological, renal, and liver functions. Written informed consent was taken from patients before enrolment. Female patients in the childbearing period must had a negative pregnancy test (serum $\beta$-HCG) and both male and female patients must employ effective contraceptive measures prior to start of until four weeks after the last dose of chemotherapy. Additionally, patients must had no concurrent malignancy.

Patients were allocated to either arms; arm A consisted of 3 cycles of neoadjuvant chemotherapy in the form of cisplatin and gemcitabine followed by radical cystectomy, arm B consisted of radical cystectomy.

\section{Settings}

The study was conducted at the Oncology Unit, Ain Shams University Hospitals, Ismailia Oncology Teaching Hospital, and National institute of Nephrology and Urology. The last 2 hospitals are run under the general organization for teaching hospitals institutes, Egypt.

\section{Study design}

The study design was shown in Table 1.

The neoadjuvant chemotherapy administration protocol for arm 1 was as follows:

1. Cisplatin: Pre-chemotherapy, normal saline 0.9\% 1 litre over 30-60 minutes, followed by Cisplatin IV in normal saline $0.9 \% 500 \mathrm{ml}$ over 1 hour. Postchemotherapy, normal saline $0.9 \% 1$ litre, with KCL (potassium chloride) $20 \mathrm{meq}, \mathrm{MgSO}_{4}$ (magnesium sulphate) $1 \mathrm{gm}$, and mannitol 30 gm over 1 hour.

2. Gemcitabine IV in normal saline $0.9 \% 250 \mathrm{~mL}$ over 30 minutes.

Cycles of chemotherapy were administered after checking CBC, renal function tests, GFR, liver function tests, bilirubin, before day 1 , and 8 of each cycle, with subsequent dose modification based on the following Table 2 (7).

\section{Evaluation}

Baseline CT thoracic-abdominal-pelvic (TAP) was performed before treatment protocol at the time of diagnosis. After the end of chemotherapy, patients underwent CT AP to evaluate chemotherapy response (Table 3). Two weeks after cycle 3 of chemotherapy, patients underwent radical cystectomy, pelvic lymphadenectomy, and urinary diversion, with transurethral resection (TUR) prior to the surgery to know the disease extent. After surgery, pathological evaluation was performed to
Table 1.

Treatment protocol of the current trial.

\begin{tabular}{|lc|}
\hline \multicolumn{2}{|c|}{ Treatment protocol } \\
\hline Arm 1 & \multicolumn{1}{c|}{ Arm 2 } \\
\hline Neoadjuvant chemotherapy; & \\
Cisplatin $\left(70 \mathrm{mg} / \mathrm{m}^{2}\right.$ Day 1$)$, Gemcitabine & \\
$\left(1250 \mathrm{mg} / \mathrm{m}^{2}\right.$ Day 1,8$) \mathrm{Q} 3$ weeks for & \\
3 Cycles & Radical cystectomy \\
\hline & \\
\hline
\end{tabular}

evaluate the chemotherapy response in Arm A, and to confirm pathological staging in Arm B.

\section{Follow-up}

After the end of the treatment protocol, patients were followed according to the NCCN guideline for follow-up after bladder cancer treatment; by regular clinic visits every 3 months for the first 2 years, then every 6 months for the following 3 years, then annually thereafter. In each visit, patients were evaluated by history taking, physical examination, laboratory investigations in the form of CBC, liver, renal functions every 3months for the first 2 years then as clinically indicated. CT TAP were done every 6 months in the first 2 years, then as clinically indicated (3).

\section{Toxicity}

Toxic effects were graded according to the National Cancer Institute Common Toxicity Criteria, version 2.0 (8). Early chemotherapy toxicities were defined as toxicities that occurred during treatment till 8-10 weeks post chemotherapy. Surgical early morbidities were defined

\section{Table 2.}

Dose modification based on hematological, and renal function results.

\begin{tabular}{|c|c|c|c|c|}
\hline \multicolumn{5}{|c|}{ 1. Hematology } \\
\hline \multicolumn{5}{|c|}{ For day 1 of each cycle: } \\
\hline \multicolumn{2}{|c|}{ Neutrophils $\left(10^{3} / \mathrm{ml}\right)$} & Platelets $\left(10^{3} / \mathrm{ml}\right)$ & & Dose \\
\hline$\geq 1$ & and & $>100$ & & $100 \%$ Both \\
\hline 0.5 to 0.99 & or & 75 to 100 & $75 \%$ & of Gemcitabine only \\
\hline$<0.5$ & or & $<75$ & & elay both drugs \\
\hline \multicolumn{5}{|c|}{ For Gemcitabine only day 8: } \\
\hline$\geq 1$ & and & $>100$ & & $100 \%$ \\
\hline 0.5 to 0.99 & or & 75 to 100 & & $75 \%$ \\
\hline$<0.5$ & or & $<75$ & & Omit \\
\hline \multicolumn{5}{|c|}{ 2. Renal function test (GFR) (ml/min) } \\
\hline GFR & \multicolumn{3}{|c|}{ Cisplatin dose } & Gemcitabine dose \\
\hline$\geq 60$ & \multicolumn{3}{|c|}{$100 \%$} & $100 \%$ \\
\hline 45 to 59 & \multicolumn{3}{|c|}{$\begin{array}{c}35 \mathrm{mg} / \mathrm{m}^{2} \mathrm{D} 1+2 \\
\text { (same pre-hydration as } 70 \mathrm{mg} / \mathrm{m}^{2} \text { dose) }\end{array}$} & $100 \%$ \\
\hline$<45$ & \multicolumn{3}{|c|}{ Delay } & Delay \\
\hline
\end{tabular}


Table 3.

Response definitions.

\begin{tabular}{|ll}
\hline Complete Response (CR) & Complete disappearance of the tumor \\
\hline Partial Response (PR) & $50 \%$ or more reduction in the size of the tumor \\
\hline Disease Progression (DP) & $25 \%$ or more increase in the size of the tumor \\
\hline Stable Disease (SD) & All other situations
\end{tabular}

as complications that occurred from day 1 postoperative till full recovery from the surgery usually 6-8 weeks postoperative. Late toxicities referred to those occurred $>10$ weeks after finish of treatment protocol.

\section{Statistical analysis}

All calculations were carried out using Prism 6 software for Windows. All analyses were carried by intention to treat. Mean and median values were used for the description of continuous data. For comparison between the 2 group characteristics, $\mathrm{T}$ test, and $\mathrm{p}$ value were used.

Table 4.

Patients and diseases characteristics of each treatment.

\begin{tabular}{|c|c|c|c|c|c|}
\hline \multirow[b]{2}{*}{ Characteristics } & \multicolumn{2}{|c|}{ Arm A } & \multicolumn{2}{|c|}{ Arm B } & \multirow[b]{2}{*}{$P$ value } \\
\hline & Number & $\%$ & Number & $\%$ & \\
\hline \multicolumn{6}{|l|}{ Age } \\
\hline $35-40$ & 1 & $3.3 \%$ & 0 & $0 \%$ & 0.1 \\
\hline $40-50$ & 20 & $67 \%$ & 14 & $47 \%$ & 0.1 \\
\hline $50-60$ & 7 & $23 \%$ & 10 & $33 \%$ & 0.1 \\
\hline $60-65$ & 2 & $6.7 \%$ & 6 & $20 \%$ & 0.03 \\
\hline Mean age & 48.9 & - & 52.3 & - & 0.04 \\
\hline \multicolumn{6}{|l|}{ Sex } \\
\hline Male & 28 & $93 \%$ & 29 & $96.7 \%$ & - \\
\hline Female & 2 & $7 \%$ & 1 & $3.3 \%$ & - \\
\hline \multicolumn{6}{|l|}{ Performance status } \\
\hline 0 & 11 & $36.3 \%$ & 9 & $30 \%$ & - \\
\hline 1 & 17 & $56.7 \%$ & 16 & $53.3 \%$ & - \\
\hline 2 & 2 & $7 \%$ & 5 & $16.7 \%$ & - \\
\hline $\begin{array}{l}\text { Performance status } \\
\text { (mean, median) }\end{array}$ & $0.7,1$ & - & $0.9,1$ & - & 0.1 \\
\hline \multicolumn{6}{|l|}{ Tumor size } \\
\hline $1-2 \mathrm{~cm}$ & 4 & $13.3 \%$ & 3 & $10 \%$ & - \\
\hline $2.1-4 \mathrm{~cm}$ & 17 & $56.7 \%$ & 15 & $50 \%$ & - \\
\hline$>4 \mathrm{~cm}$ & 9 & $30 \%$ & 12 & $40 \%$ & - \\
\hline Mean tumor size & 3.7 & - & 3.8 & - & 0.1 \\
\hline \multicolumn{6}{|c|}{ Histopathological grade } \\
\hline 1 & 3 & $10 \%$ & 2 & $6.7 \%$ & - \\
\hline 2 & 7 & $23.3 \%$ & 6 & $20 \%$ & - \\
\hline 3 & 20 & $66.7 \%$ & 22 & $73.3 \%$ & - \\
\hline Grade mean, median & $2.5,3$ & - & $2.6,3$ & - & 0.1 \\
\hline \multicolumn{6}{|l|}{ Tumor stage } \\
\hline pT2NO & 6 & $20 \%$ & 8 & $26.7 \%$ & - \\
\hline pT3NO & 18 & $60 \%$ & 18 & $60 \%$ & - \\
\hline pT4aNO & 6 & $20 \%$ & 4 & $13.3 \%$ & - \\
\hline Tumor stage median & T3 & & T3 & & 0.07 \\
\hline
\end{tabular}

Overall survival (OS) and progression-free survival (PFS) for each arm were analyzed by the Kaplan-Meier method. Further, they were compared using the log rank and Wilcoxon tests. OS was measured from the time of randomization till death from bladder cancer or the last follow-up visit. PFS was measured from the time of randomization till relapse, or the last follow-up visit.

Log rank approach and hazard ratio were used to examine the effects of pre-specified prognostic factors including age, tumor size, pathological staging, and pathological response to neoadjuvant chemotherapy on the 3 year OS. P value was significant at $\leq 0.05$.

\section{RESULTS}

Between September 2009 and April 2014, 60 patients were enrolled in the current study. 30 patients were assigned to each treatment arm. All patients fulfilled eligibility criteria for enrolment in the current study. The mean age was 50.6 years (range $30-65$ years). 56 patients were males, and 4 were females (93.3\%, 6.7\% respectively). The median performance status was 1 (range $0-2$ ). The mean tumor size was $3.75 \mathrm{~cm}$ (range 1.8-5.5 cm). 12 patients had stage T2NO, 38 had stage T3NO, and 10 had stage T4aNO. (20\%, 63.3\%, 16.7\% respectively) (Table 4).

\section{Treatment Protocol}

For Arm A:

All arm A patients received neoadjuvant chemotherapy, and a total of 89 cycles were performed. Mean chemotherapy cycles were 3 (range 2-3). To evaluate treatment response, CT AP was checked at mean time of 2.2 weeks after cycle 3 (range 1-3 weeks).

\section{Neoadjuvant chemotherapy response}

Among the 30 patients enrolled, CR was achieved in 6 patients (20\%), PR was observed in 16 patients (53.3\%), and SD was in 6 patients $(20 \%)$. The remaining 2 patients had DP (6.7\%).

\section{Surgery}

Radical cystectomy, pelvic lymphadenectomy, and urinary diversion were performed in 58 out of the 60 patients. For arm A patients, 28 underwent cystectomy, the remaining 2 refused surgery and lost follow-up. Of them, 19 had neobladder, 7 ileal conduit, and 2 underwent cutaneous urinary diversion. Surgery was done at a mean of 2 weeks after cycle 3 chemotherapy (range 1021 days). The mean admission time for surgery was 8.3 days (range 7-14 days). The average blood loss was 900 $\mathrm{ml}$ (range 500-2000 ml).

Pathological evaluation after cystectomy was done in all the 28 patients. R0 was achieved in all except 2 patients who had Rl disease. PCR (pathological complete remission) (pT0) was achieved in 10 patients (35\%), pPR (pathological partial remission) in 12 patients (43\%) (8 pT1, and 4 patients pT2). The remaining 6 had SD (stable disease) (22\%) (2 pT2, 3 pT3, and 1 pT4). The mean number of dissected pelvic lymph nodes was 15 (range 7-20): 4 patients had positive lymph node biopsies. For arm $\mathrm{B}$, all the 30 patients underwent cystectomy. Of them, 18 underwent neobladder, 6 ileal conduit, 5 cuta- 
neous reservoir, and 1 had cutaneous urinary diversion. Surgery was done at mean of 3 weeks after diagnosis (range 17-30 days). The mean admission time for surgery was 7 days (range 6-10 days). The average blood loss was $850 \mathrm{ml}$ (range 450- $2000 \mathrm{ml}$ ). Pathological evaluation was done in all the 30 patients. R0 was achieved in 26 patients. The remaining 4 had Rl disease. Pathological staging was pT2, pT3, and pT4a in 7, 18, and 5 patients respectively. The mean number of dissected pelvic lymph nodes was 13 and 6 patients had positive lymph node biopsies.

Adjuvant chemotherapy was given to 4 patients from arm A for positive lymph node in the form of cisplatin and gemcitabine for 3 cycles.

Additionally, 13 patients from arm B received adjuvant chemotherapy ( 6 positive lymph nodes, $2 \mathrm{Rl}$, and 5 pT4a) in the form of either cisplatin and gemcitabine, or carboplatin and gemcitabine. (6, and 7 patients respectively), for a mean of 4 cycles (range 3-6).

\section{Survival data}

The 3 year OS for arm A, and arm B were 60\%, 50\% respectively (Figure 1). The median OS for arm A was $36+$ months and that for arm B was 32.5 months. The 3 year PFS for arm A and B were $57 \%$ and $43 \%$ respectively (Figure 2). The median PFS for arm A was 36+ months and that for arm B was 28 months. During the 36 months follow-up period, 11 patients died from arm A ( 9 bladder cancer relapses, 1 cardiovascular cause, and 1 unknown cause). For arm B, 15 patients died (12 bladder cancer relapses, 2 pulmonary embolism, and 1 unknown cause). During the 36 months follow-up period, 12 patients from arm A relapsed, (9 locoregional, and 3 metastatic), and 17 relapsed from arm B (11 locoregional and 6 metastatic).

For subgroup analysis, the 3 year OS for arm A patients who were $<60$ y.o was $64 \%$, and for those $>60$ years was $50 \%$. The 3 year OS for arm B patients who were $<$ 60 y.o was $54 \%$, and for those $>60$ years was $17 \%$. (P value 0.02 , chi square 5.5 ).

The 3 year OS for arm A T2 patients was $83 \%$, and for T3-T4a patients was $54.5 \%$. The 3 year OS for arm B T2

\section{Figure 1.}

The 3 year OS for the study groups

$P$ value $=0.05$, chi square $=3.66$.

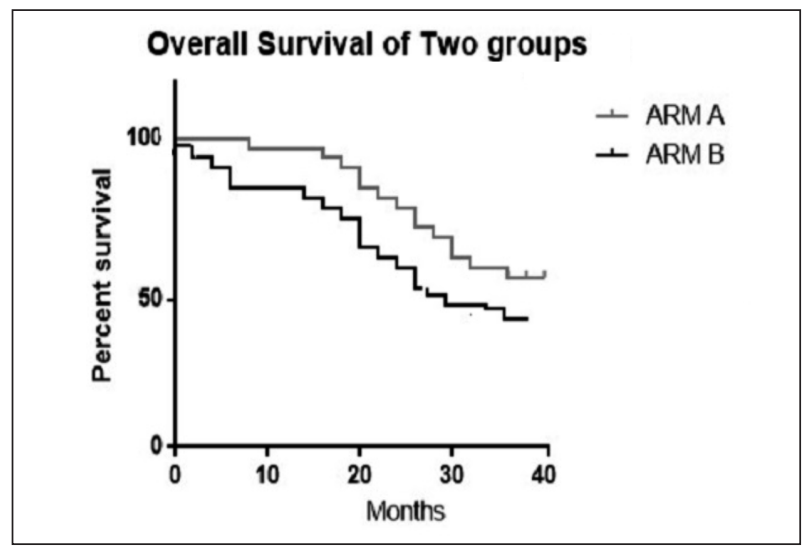

Figure 2.

The 3 year PFS for the study groups

$P$ value $=0.02$, chi square $=4.88$.

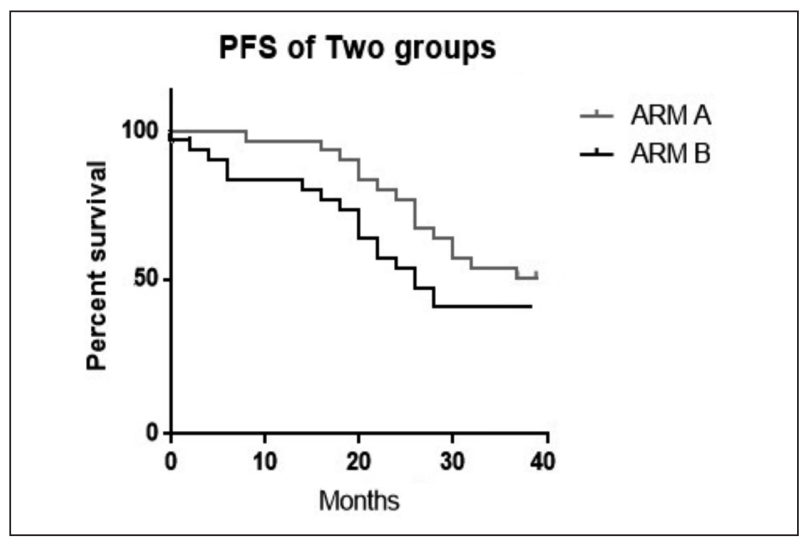

Table 5.

The 3 year OS for each treatment arm categorized by the prognostic factors.

\begin{tabular}{|lcccc|}
\hline & Arm A (\%) & Arm B (\%) & P value & Hazard ratio \\
\hline 1. Age & & & & \\
\hline$<60$ years old & 64 & 54 & 0.02 & 1.8 \\
$>60$ years old & 50 & 17 & 0.2 & 1.1 \\
\hline 2. Pathological stage & & & & \\
\hline pT2 & 83 & 75 & 0.05 & 1.6 \\
PT3-T4a & 54.5 & 41 & 0.08 & 1.55 \\
\hline 3. Tumor size & & & & \\
\hline$<4 \mathrm{~cm}$ & 70 & 61 & 0.1 & 2.0 \\
$>4 \mathrm{~cm}$ & 37.5 & 33 & 0.09 & 1.2 \\
\hline
\end{tabular}

patients was $75 \%$, and those with T3-T4a was $41 \%$ ( $P$ value 0.01 , chi square 5.7). The 3 year OS for arm $A$ patients who have tumors $<4 \mathrm{~cm}$ was $70 \%$, and for those $>4 \mathrm{~cm}$ was $37.5 \%$. The 3 year OS for arm B patients who

Figure 3.

The Kaplan-Meier survival curve for arm A patients grouped by their pathological response to neoadjuvant chemotherapy. $P$ value $=0.3$.

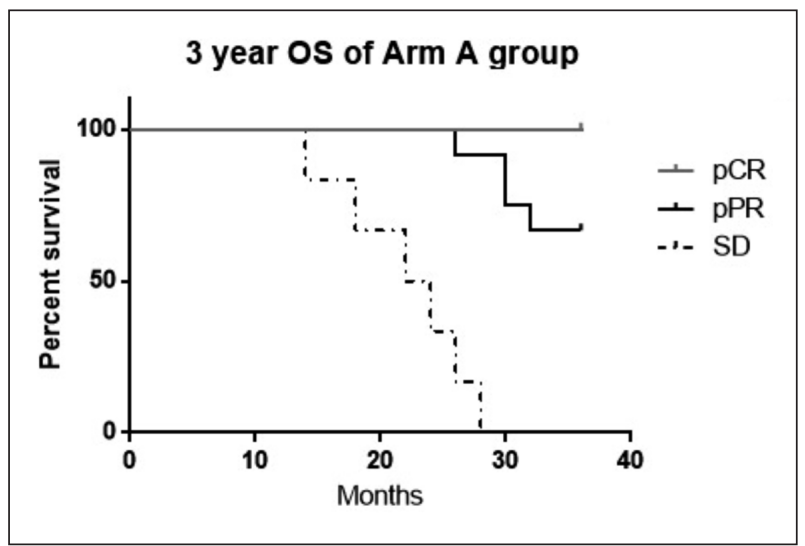


have tumors $<4 \mathrm{~cm}$ was $61 \%$, and for those $>4 \mathrm{~cm}$ was $33 \%$ (P value 0.1 , chi square 2.2 ).

For the 3 year follow-up for arm A patient, all those who achieved pCR were still alive (100\%). Of them, only 1 patient relapsed (10\%) after 26 months of treatment. For those who achieved pPR, 8 patients were still alive (67\%), and 3 died from cancer recurrence. Further, 1 patient recurred, and was still alive. For those who achieved SD pathologically, all of them died. (P value 0.0001 , chi square 23.3) (Figure 3).

\section{Toxicity profile}

Chemotherapy side effects:

For arm A, among the 89 chemotherapy cycles given, dose reduction was done in $10 \%$ of cycles for leuconeutropenia. Treatment delay was in $10 \%$ of cycles. The mean delay time was 1 week (range 1-2 weeks). Cisplatin was replaced by Carboplatin in the last 2 cycles for 1 patient for persistent low GFR. Treatment was stopped in the last cycle in 1 patient for persistent urinary tract infection (UTI), and poor general condition.

Table 6.

Grade 3-4 side effects, and their percentage for arm A group.

\begin{tabular}{|lcc|}
\hline Side effect & \multicolumn{2}{c|}{ Arm A } \\
\hline Leuconeutropenia & Grade 4 (\%) & Grade 3 (\%) \\
Anemia & 12.2 & 2.2 \\
Thrombocytopenia & 2.2 & - \\
Febrile neutropneia & 17.8 & - \\
Nausea, Vomiting & 2.2 & - \\
Mucositis & 4.4 & - \\
Others (UTI) & 5.5 & - \\
\hline
\end{tabular}

Table 7.

The early surgical complications in both arms and their percentage.

\begin{tabular}{|lcc|}
\hline Side effect & \multicolumn{2}{c|}{ Arm A } \\
\hline Leuconeutropenia & Grade 4 (\%) & Grade 3 (\%) \\
Anemia & 12.2 & 2.2 \\
Thrombocytopenia & 2.2 & - \\
Febrile neutropneia & 17.8 & - \\
Nausea, Vomiting & 2.2 & - \\
Mucositis & 4.4 & - \\
Others (UTI) & 5.5 & - \\
\hline
\end{tabular}

No deaths occurred related to treatment in each group (Table 6-7).

Table 6 summarize grade 3-4 side effects, and their percentage for arm A group.

Table 7 showed the early surgical complications in both arms and their percentage.
All patients who developed early surgical complications recovered smoothly.

\section{Late toxicities}

During the 36 months follow-up period, 1 patient from arm A died by congestive cardiac failure at 30 months. Further, 1 patient developed impaired renal function from repeated UTI and he still alive and did not require dialysis, yet. For arm B patients, 2 patients died from pulmonary embolism (18, 27 months).

\section{Discussion}

The role of neoadjuvant chemotherapy in urinary bladder cancer was strongly encouraged in stages pT2-T4a bladder cancer, based on 3 important trials, including that of Griffiths et al., 2011, Vale et al., 2003, and Winquist et al., 2004 (4-6).

However, there are still controversies about its impact on survival. Griffiths et al., 2011 (4), reported that, neoadjuvant chemotherapy (MVAC) slightly improved survival by $6 \%$ over 5 years. However, this slight improvement in survival can be attributed to their use of MVAC regimen, which was associated with significant toxicity profile (9). The aim of neoadjuvant chemotherapy is to achieve tumor downstaging, improved respectability, and better survival $(10,11)$.

Neoadjuvant chemotherapy is considered better than adjuvant chemotherapy in relation to its tolerability, and patients usually receive adequate cycles of neoadjuvant chemotherapy with effective doses. It is still unclear which neoadjuvant chemotherapy regimen offers the best results. In patients with advanced or metastatic TCC the combination of gemcitabine and cisplatin achieved comparable survival results with MVAC, and was associated with less toxicity (10).

For the current study, although, there was no clear epidemiological trials that reported definite disease characters among the Egyptians, our patient cohort, and distribution were nearly equivalent to that of Fedewa et al., 2009 (1), which showed the incidence of bladder cancer in the Nile delta region of Egypt. Further, our patients were properly randomized with no significant differences in patient characters between the 2 arms.

There were non-statistically significant differences between the 2 groups in relation to age, tumor size, and performance state in favor of arm A. However, the difference in age was statistically significant for the group of patients $>60$ years.

This was explained by the fact that the investigators tried to avoid giving chemotherapy in older ages to avoid its long term complications, as well as to avoid delay in the definitive surgery especially with larger tumor sizes in this group.

In the current trial, the primary end point was survival for neoadjuvant chemotherapy as compared with the standard treatment that is cystectomy. We believed that survival benefit had to be the main concern for trials like ours that treated cancers with curative intent. However, the follow-up period was not long enough to show clear survival benefit over a long period of time. The researchers of the current trial used to define survival in 
relation to several subgroup analysis for better evaluation, and to define which subgroup would benefit most from treatment protocol. Further, the researchers planed to make an updated report for the survival benefit, and toxicity profile after 5 years, and hopefully after 10 years follow-up periods.

For the diversion procedures, although orthotopic diversion was the standard in our institutes, the authors tried to avoid the metabolic complications of orthotopic diversion especially after neoadjuvant cisplatin containing regimen. The current study clearly showed survival benefit for neoadjuvant cisplatin and gemcitabine combination over radical cystectomy. Further, the survival was better for each subgroup for arm A over arm B.

The current study showed better surgical resectability for arm A patients, when compared with arm B patients. Arm A patients had higher R0 number over arm B (100\% vs $86 \%$ respectively). Further, neoadjuvant chemotherapy achieved tumor downstaging in $78 \%$ of patients.

For toxicity profile, neoadjuvant chemotherapy was tolerated well, and was associated with mild grade 3-4 toxicities. Surgery after chemotherapy was associated with very comparable side effects with that of arm B. Bleeding complication were relatively higher among the group who received neoadjuvant chemotherapy.

For the delayed side effects, the incidence of them was nearly equivalent between the 2 treatment groups. Further, reports with relatively longer follow-up durations are needed before confirming that point.

\section{Conclusion}

Neoadjuvant chemotherapy before cystectomy achieved better survival results, surgical respectability, and nearly equivalent toxicities when compared with radical cystectomy in the management of stage II, and III urothelial bladder cancer.

\section{References}

1. Fedewa SA, Soliman AS, Ismail K, et al. Incidence analyses of bladder cancer in the Nile delta region of Egypt. Cancer Epidemiol. 2009; 33:176-181.

2. Edge SB, Byrd DR, Compton CC, et al. Eds: AJCC Cancer Staging Manual. 7th ed. Urinary bladder. New York, NY: Springer, 2010, pp 497-505.

3. NCCN clinical practice guidelines in oncology, bladder cancer, version 1, 2014, http://www.nccn.org/professionals/physician_gls/ f_guidelines. asp\#bladder.

4. Griffiths $G$, Hall R, Sylvester R, et al. International phase III trial assessing neoadjuvant cisplatin, methotrexate, and vinblastine chemotherapy for muscle-invasive bladder cancer: long-term results of the BA06 30894 trial. J Clin Oncol. 2011; 29:2171-2177.

5. Vale C, Advanced Bladder Cancer Meta-analysis Collaboration MRC Clinical Trials Unit: Neoadjuvant chemotherapy in invasive bladder cancer: a systematic review and meta-analysis. Lancet. 2003; 361:1927-1934.

6. Winquist E, Kirchner TS, Segal R, et al. Neoadjuvant chemotherapy for transitional cell carcinoma of the bladder: a systematic review and meta-analysis. J Urol. 2004; 171:561-569.

7. BCCA Protocol Summary for Palliative Therapy for Urothelial Carcinoma Using CISplatin and Gemcitabine. http://www.bccancer. bc.ca/NR/rdonlyres/A2451205-5C13-433C-AADE 2E0DC1519D71/67037/GUAVPG_Protocol_1Nov2013.pdf, 2013.

8. Common Toxicity Criteria (CTC) Version 2.0, http://www.eortc. be/services/doc/ctc/ctcv20 4-30-992.pdf-Published April 30, 1999.

9. Calabrò F, Sternberg CN. Localized and locally advanced bladder cancer. Curr Treat Options Oncol. 2002; 3:413-28.

10. von der Maase H, Sengelov L, Roberts JT, et al. Long-term survival results of a randomized trial comparing gemcitabine plus cisplatin, with methotrexate, vinblastine, doxorubicin plus cisplatin in patients with bladder cancer. J Clin Oncol. 2005; 23:4602-4605.

11. Herr HW, Dotan Z, Donat SM, Bajorin DF. Defining Optimal Therapy for Muscle Invasive Bladder Cancer. J Urol. 2007; 177:437-443.

\section{Correspondence}

Mohammed A Osman, MD (Corresponding Author) mmoneam@hotmail.com

Oncology Consultant, General Organization for Teaching Hospitals Institutes Egypt

Ayman M Gabr, MD

keshta64@gmail.com

Urology Consultant, National Institute of Urology \& Nephrology, Egypt 